\title{
Range-free localization using expected hop progress in anisotropic wireless sensor networks
}

Wu Wen ${ }^{1,2^{*}}$, Xianbin Wen ${ }^{1,2^{*}}$, Liming Yuan ${ }^{1,2}$ and Haixia $X u^{1,2}$

\begin{abstract}
Accurate localization of nodes is one of the key issues of wireless sensor network (WSN). A localization algorithm using expected hop progress (LAEP) has been successfully applied in isotropic wireless sensor networks. However, range-free LAEP cannot be directly used for anisotropic WSNs because anisotropic problems limit the applicability of multi-hop localization. In order to solve the problem, an improved localization algorithm is proposed to reduce the localization error. In this paper, we adapt the expected hop progress to anisotropic WSNs by considering both hop count computation and anchor selection. Then, particle swarm optimization algorithm is introduced to improve the positioning accuracy. The experimental results demonstrate that our algorithm has better higher precision than do state-of-the-art algorithms. Even for isotropic WSNs, our algorithm always outperforms its counterparts.
\end{abstract}

Keywords: Anisotropic wireless sensor networks, Range-free, Multi-hop, Expected hop progress, Particle swarm optimization

\section{Introduction}

Wireless sensor networks (WSNs) are an emerging technology that has potential applications in various fields, such as healthcare, surveillance, astronomy, military and agriculture [1-4]. Most of these applications require knowledge of the exact locations of the sensor nodes used to sense the data. In the absence of such information, data may not be useful for users. Therefore, the precise localization of sensors is a critical requirement in WSNs [5].

The localization issue in WSNs can be resolved by using the global positioning system (GPS) with each sensor node, but this is not favourable due to energy, cost and size issues. An efficient and better alternative is required to localize the sensor nodes. Various non-GPS-based localization algorithms have been used, which are categorized into range-based $[6,7]$ and range-free $[8,9]$ algorithms. Although the range-based algorithms are more accurate than the range-free localization algorithms, they

\footnotetext{
*Correspondence: 15566524241@163.com; xbwen317@163.com

${ }^{1}$ Key Laboratory of Computer Vision and System, Ministry of Education,

Tianjin University of Technology, Tianjin 300384, China

Full list of author information is available at the end of the article
}

require a very high cost. Unlike range-based algorithms, range-free algorithms, which rely on the network connectivity to estimate the positions of regular nodes without any extra hardware supporting, are more power-efficient and do not require additional hardware. At present, most researchers focus on isotropic WSNs. Wireless sensor networks are mostly applied to complex environments where there are obstacles and holes, in which case they are called anisotropic wireless sensor networks (AWSNs). In this case, when the line connecting two nodes passes these obstacles, the shortest paths between anchor nodes and regular nodes are likely to be curved and its length may be estimated much larger than corresponding Euclidean distance. Therefore, position estimation is inaccurate.

DV-Hop [10], Amorphous [11], MDS-MAP [12] and APIT [13] are examples of early range-free localization schemes that are well suited for isotropic wireless networks (i.e. where obstacles do not exist). However, the distance estimation accuracy of these methods is severely degraded in anisotropic networks, resulting in unacceptable overall localization errors. To solve this problem, a few new range-free algorithms are proposed for tolerating erroneous distance estimates in AWSNs. 
The proximity distance mapping (PDM) [14] algorithm replaces the average hop distance with a proximity-distance mapping matrix in estimating the distances between nodes and anchors. Substantial topological information can be preserved by the mapping matrix. The pattern-driven scheme (PDS) [15] algorithm applies various distance estimation algorithms for anchors based on their exhibited patterns. Next, the anchor supervised [16] algorithm is presented. In this approach, every anchor selects a set of reliable anchors for which distance estimates can be accurately obtained. Later, a location algorithm that uses the expected hop progress (EHP) was proposed in $[17,18]$. A modified EHP approach is obtained by redefining a new cumulative distribution function (CDF) and achieves satisfactory localization results [19]. The algorithm depends not only on the communication radius of the anchors, but also on the communication radius of the inter-nodes, which is closer to the real Euclidean distance between any two nodes. Recently, Farrukh Shahzad proposed a scheme, called DV-maxHop [20], that can achieve similar or better performance by just introducing a control parameter MaxHop in the first phase of the DV-Hop algorithm. However, most of these methods do not achieve better positioning accuracy or achieve better accuracy at the expense of high computational or communication overheads. Therefore, a low-cost and high-precision algorithm for anisotropic WSNs is necessary.

In this paper, we propose a novel range-free localization algorithm based on the modified EHP and a particle swarm optimization algorithm (PSO) [21] that is tailored for anisotropic WSNs. First, we assume that the degree of irregularity (DOI) of the communication radius is equal to zero. Then, the distance from regular nodes to reliable anchors can be estimated precisely by introducing a control parameter, MaxHop. The reliable anchors are properly chosen following a new reliable anchor selection strategy. Next, we use the mathematical expectation of CDF to estimate the distances between the regular nodes and the reliable anchors. Finally, the PSO algorithm is used for localization optimization.

The organization of this paper is as follows. Section 2 presents the localization model of multi-hop AWSN. Then, the proposed range-free localization algorithm and PSO algorithm are proposed in this section. The simulation results and performance evaluations are analysed in Section 3. Finally, Section 4 concludes the paper.

\section{Methodology}

\subsection{Network model and overview}

For our study, wireless nodes are deployed in a two-dimensional (2-D) square area. In the anisotropic network case, there are one or two rectangular structures or holes where nodes cannot be deployed. As shown in Fig. 1, under such anisotropic terrain conditions, $N$ sensor nodes are uniformly deployed in a square area $S$ that contains a rectangular obstacle, which form a C-shaped network topology. The signal does not pass through these obstacles. All nodes are assumed to have the same transmission capability, except when we consider the effect of DOI during simulation. Each node can directly communicate with any other node in the disc, with that node as the centre and radius. Each anchor node is equipped with a GPS receiver, and they are aware of their positions. The other nodes, called regular nodes, do not know their own positions [22, 23].

The major symbols used in this paper are listed as follows:

$N=$ the total number of all nodes;

$N_{a}=$ the number of anchor nodes (ANs);

$N_{u}=$ the number of unknown nodes (UNs);

$R=$ the communication range or radius of each

node $(\mathrm{m})$;

$\lambda=$ the node density in the monitoring area;

area $(k, R)=$ the $k$-th node's coverage area with the $k$-th sensor as the centre and radius $R$.

In multi-hop AWSN localization, the goal is to estimate the locations of all UNs by using ANs and partial information of the distances between various pairs of ANs and UNs. We suppose that the $i$-th anchor node broadcasts data packet containing its position and the $j$-th regular node receives the data packet through multi-hop communication. Then, we employ the shortest path method to obtain a possible path between a source sensor and a destination sensor with the

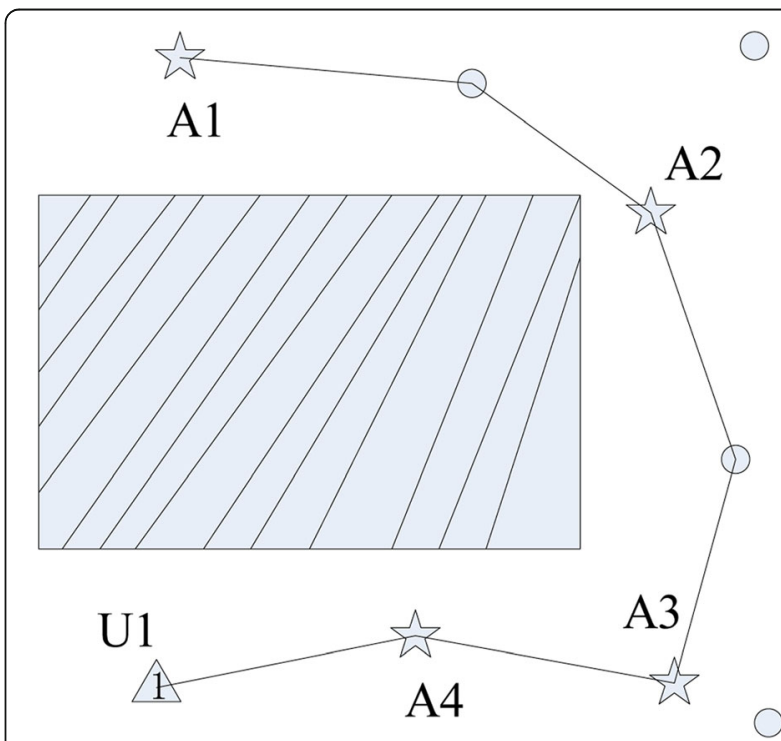

Fig. 1 Network model 
minimum number of hops. Let $n_{i j}$ be the number of hops between the $i$-th anchor and the $j$-th regular node. The distance $\hat{d}_{i-j}$ from the $j$-th regular node to the $i$-th anchor is estimated as follows [24]:

$$
\hat{d}_{i-j}=n_{i j} \bar{h}_{s}
$$

where $\bar{h}_{s}$ is a predefined average hop distance. To a large extent, this distance estimation approach relies on the high density of WSNs.

Although heuristic and analytical algorithms are proven to be sufficiently accurate in isotropic WSNs, their accuracies are not optimal in anisotropic WSNs. It is very likely that the shortest path between an anchor node and a regular node is curved in an AWSN, thereby resulting in an overestimation of the hop count between these two nodes. According to Fig. 1, the hop size between nodes $A_{1}$ and $U_{1}$ is six hops; however, the number of hops between them is far smaller due to obstacles. The larger the hop size estimation errors are, the greater the distance estimation errors are, and consequently, the less accurate the localization is. To solve this problem, we propose a novel localization algorithm that is based on new reliable anchor selection strategy. We introduce a parameter MaxHop in the first phase of our algorithm. The algorithm ignores the information if the hop count is greater than MaxHop. In the anisotropic network, when two nodes locate at two ends of an obstacle group, we ignore the farther anchor which will cause a detoured path, and consequently, the shortest path between two nodes will not be curved. Then, the EHP method is adopted to calculate the average hop distance. In Fig. 1, regular node $U_{1}$ will select $\mathrm{A}_{4}$ and $\mathrm{A}_{3}$ as reliable anchors. We can use the average hop distance to make the distance calculation among regular node $U_{1}$ and reliable anchors more precise in AWSNs. In the next section, we derive the expression for $\bar{h}_{s}$ that is exploited later in our algorithm.

\subsection{The proposed algorithm and its analysis}

\subsubsection{Hop distance derivation using the EHP approach}

The EHP algorithm is based on an accurate analysis of hop progress in WSNs. We can derive the distance between any two nodes through expected hop progress. In the network model with uniform sensor transmission range and arbitrary node density, the expected hop progress of each hop in this network is the same. Therefore, the expected hop progress can be used to replace the average hop distance. In this section, for the sake of clarity, we discuss only two-hop communication in which the $i$-th node communicates with the $j$-th node through an intermediate node $k$. For clarity, let the random variables $X$ and $Z$ represent the distances $d_{i-j}$ and $d_{i-k}$, respectively. Then, we use the expectation of $Z$ to represent the average hop distance, as shown in formula (2) [25].

$$
\bar{h}_{s}=E\{Z\}
$$

To obtain a more accurate value for $\bar{h}_{s}$, we use the conditional cumulative distribution function $(\mathrm{CDF}) F_{Z}$ । ${ }_{X}(z)=P(Z \leq z \mid x)$ of $Z$ to represent the average hop distance. In Fig. 2, if $Z \leq z$ is guaranteed, then there are no nodes in the dashed area $A$. We define the conditional CDF in formula (3).

$$
F_{Z \mid X}(z)=P(Z \leq z \mid x)=P\left(A_{0} \mid Q_{1}\right)
$$

where $Q_{1}$ is the potential forwarding area wherein the $k$ th node communicates directly with the $j$-th and $i$-th nodes and $P\left(A_{0} \mid Q_{1}\right)$ is the probability that event $A_{0}$ occurs given event $Q_{1} . A_{0}$ indicates that there are no nodes in the dashed area $A$, and $Q_{1}$ indicates that there is at least one node in the potential forwarding area $Q$, which depends on communication radius $R$. The potential forwarding area $Q$ is given by formula (4).

$$
Q=\operatorname{area}(i, R) \cap \operatorname{area}(j, R)=A \cup B
$$

The probability of there being $K$ nodes in area $A$ follows the binomial distribution $X \sim B(N, p)$, where $p=A$ / $S$. For relatively large $N$ and small $p, B(N, p)$ can be accurately approximated by a Poisson distribution, as shown in formula (5).

$$
F_{Z \mid X}(Z)=e^{-\lambda A}
$$

where $\lambda=\mathrm{N} / \mathrm{S}$ is the average node density of the network. We can easily calculate the area of $A$ using geometrical properties and trigonometric transformations. It is straightforward to show that:

$$
\begin{aligned}
A= & R^{2}\left(\theta+\theta^{\prime}+\theta_{z}^{\prime}-\frac{\sin (2 \theta)+\sin \left(2 \theta^{\prime}\right)+\sin \left(2 \theta_{z}^{\prime}\right)}{2}\right) \\
& -z^{2}\left(\theta_{z^{-}} \frac{\sin \left(2 \theta_{z}\right)}{2}\right)
\end{aligned}
$$

where

$$
\left\{\begin{array}{c}
\theta=\arccos \left(x^{2} /(2 \mathrm{R} x)\right) \\
\theta^{\prime}=\arccos \left(x^{2} /(2 R x)\right) \\
\theta_{z}=\arccos \left(\left(z^{2}-R^{2}+x^{2}\right) /(2 z x)\right) \\
\theta_{z}^{\prime}=\arccos \left(\left(R^{2}-z^{2}+x^{2}\right) /(2 R x)\right)
\end{array}\right.
$$

Combined with the above analysis, the average hop distance of the whole network can be derived as follows [26]: 


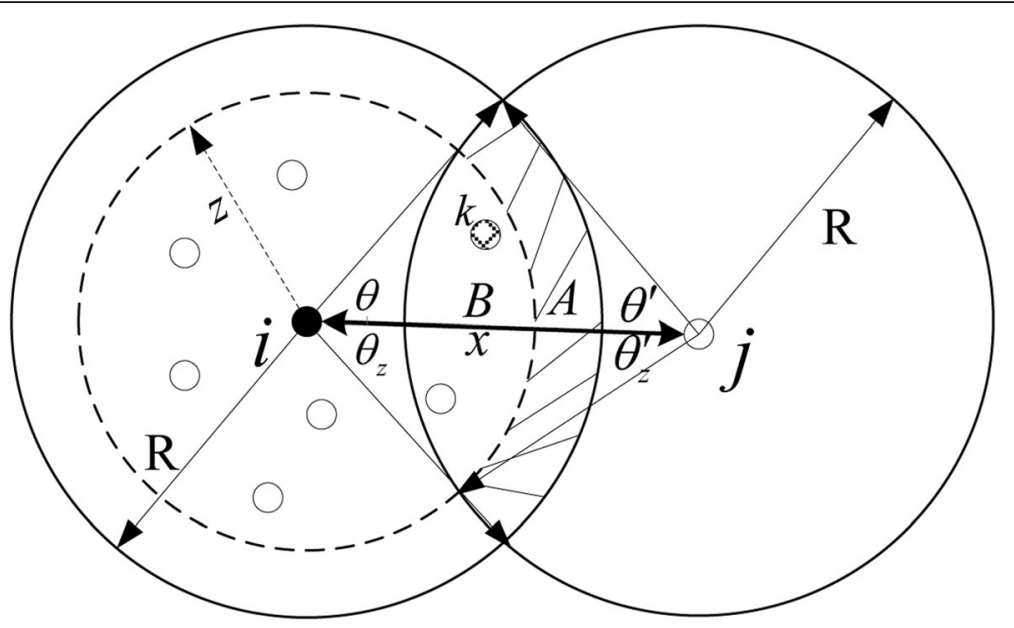

Fig. 2 Distance analysis

$$
\begin{aligned}
\bar{h}_{s}= & E_{x}\left(\alpha\left(1-F_{Z \mid X}(\alpha)\right)+E_{x}\left(\int_{\alpha}^{R}\left(1-F_{Z \mid X}(z)\right) d_{z}\right)\right. \\
= & \int_{R}^{2 R} \alpha\left(1-F_{Z \mid X}(\alpha)\right) f_{X}(x) d x+\int_{R}^{2 R}\left(\int_{\alpha}^{R}\left(1-F_{Z \mid X}(z)\right) d z\right) \\
& f_{X}(x) d x
\end{aligned}
$$

where $\alpha=x-R$ and $f_{X}(x)$ is the pdf of $X$, which can be substituted by $1 / R$, because $f_{X}(x)$ is a uniform random variable over $[R, 2 R]$. If $R$ is fixed, it is very likely that the per-hop distance increases if the number of nodes located inside $Q$ increases. According to formula (8), $\bar{h}_{s}$ can be derived if the node density and the transmission range are given. In this way, a more accurate average hop distance can be easily obtained in AWSNs through finite integrals.

When $\bar{h}_{s}$ is determined, each unknown node collects the gradients of all its neighbouring nodes relative to an anchor node and uses the local mean to replace the hop counts. As shown in formula (9), the Amorphous method is adopted to calculate the minimum hops from node $j$ to anchor nodes and the minimum hops is reduced by 0.5 through previous experimental statistics [7].

$$
S_{j}=\frac{\sum_{j^{\prime} \in \operatorname{neighbours}(j)} h_{j}+h_{j^{\prime}}}{|\operatorname{neighbours}(j)|+1}-0.5
$$

where $h_{j}$ and $h_{j^{\prime}}$ are the smallest hop counts of the unknown node $j$ and the neighbouring node of node $j$, respectively, to the anchor node; neighbours $(j)$ is the set of all neighbouring nodes of node $j$.

\subsubsection{Anchor selection strategy}

In general, the greater the hop count between two nodes, the higher the distance estimation error in the AWSN. To solve this problem, we propose a new reliable anchor selection strategy in which a hop size threshold is set; we call this parameter MaxHop [20]. When a node receives the position of any anchor with its hop count, the algorithm ignores the information if the hop count is greater than MaxHop; consequently, the information is not propagated further. This algorithm reduces the superposition of this cumulative error, improves the positioning accuracy, and reduces the network traffic. To obtain better positioning accuracy and low overheads, the threshold MaxHop should be set as close as possible to the smallest integer value on the basis of the successful positioning of all nodes.

The size of the hop threshold MaxHop is mainly determined by the connectivity and anchor node density of the network. Its expression can be derived as follows [27]:

$$
\text { MaxHop }>\frac{1}{R} \sqrt{\frac{S T}{N_{a} \pi}}
$$

where $R$ is the communication radius of the node, $N_{a}$ is the total number of anchor nodes in the network, $S$ is the acreage of the network area, and $T$ is the minimum number of anchor nodes that are required for locating unknown nodes. In practical engineering, it is difficult to achieve a highly uniform distribution of nodes. To improve the positioning rate and positioning accuracy of the nodes, the value of $N$ should be increased appropriately to ensure the overall coverage of the network. In the next section, we conduct 
an experiment to further explore the appropriate value of the threshold MaxHop. Then, we use the corrected hop counts and formula (1) to estimate the distances between the unknown nodes and the anchor nodes.

\subsubsection{Particle swarm optimization}

PSO is an optimization algorithm that was proposed by American researchers in 1995 for mimicking the collective behavior of intelligent animals. The PSO searches the space of a fitness function by adjusting the trajectory of individual particles. In other words, the purpose of this algorithm is to find the global optimum until the fitness function no longer improves. We use the position of the best particle after a fixed number of iterations as the estimated location of the target. The advantages of this algorithm are as follows: provides fast convergence and high accuracy, is easy to implement and requires few parameters [28, 29]. In most applications, PSO is used as an evolutionary computation technology. The main steps of the PSO algorithm are as follows:

1. Initialize $M$ particles. The particle iteration number is expressed as $t$. In the search space, the position and velocity are expressed as formula (11).

$$
\begin{aligned}
& X_{a}^{t}=\left(x_{a 1}^{t}, x_{a 2}^{t}\right) \\
& V_{a}^{t}=\left(v_{a 1}^{t}, v_{a 2}^{t}\right)
\end{aligned}, a=1,2, \ldots, M
$$

where $X_{a}^{t}$ is the current position of particle $(a), V_{a}^{t}$ is the velocity vector of particle $a$, and $M$ is the total number of particles.

2. Select a suitable fitness function. It is used to judge the individuals in the population.

3. Update the velocity and position of the particle. At each iteration number $(t+1)$, the velocity and position of particle $(a)$ are updated according to the following two equations:

$$
\begin{gathered}
V_{a}^{t+1}=V_{a}^{t}+c_{1} \times\left(\text { pbest }_{a}-X_{a}^{t}\right)+c_{2} \times\left(\text { gbest }_{a}-X_{a}^{t}\right) \\
X_{a}^{t+1}=X_{a}^{t}+V_{a}^{t+1}
\end{gathered}
$$

where pbest is the individual extremum, gbest is the global extremum, and $c_{1}$ and $c_{2}$ are cognition coefficients, which are random values in the range $(0,2)$.

4. Judge whether the termination conditions are satisfied. If the conditions are satisfied, the cycle is terminated; otherwise, step 2 and step 3 are repeated.

5. Select the global extremum. When the maximum number of iterations is reached, the value of gbest that is selected by the fitness function is used as the estimated coordinates of the unknown nodes.

PSO is widely used in various fields, but less research has been conducted on the particle swarm optimization algorithm in AWSNs. The localization problem can be regarded as an optimization problem, and we can use PSO to correct the estimated positions. Therefore, we mainly study the optimization effect of PSO on the positioning accuracy under the AWSN environment. In this process, it is very important to determine the fitness function. PSO begins with a group of random particles and finds the optimal solution through an iterative process. In the iterative process, the particle updates itself by tracking "two optimal solutions": one is the optimal solution obtained by the particle, namely, the local best position, and the other is the optimal solution obtained by the entire particle group, namely, the global best position. The position of the optimal particle is the coordinate position of the unknown node. Thus, the selection of the fitness function will directly affect the positioning accuracy. The distance error between the anchor nodes is usually used as a fitness function, as shown by formula (13).

$$
\begin{gathered}
\text { fitness }(x, y)=\sum_{i=1}^{N_{a}} \eta f_{i}^{2} \\
f_{i}=\left|\sqrt{\left(x-x_{i}\right)^{2}+\left(y-y_{i}\right)^{2}}-\hat{d}_{i-j}\right|
\end{gathered}
$$

where $x$ and $y$ are the coordinates of the particle, $x_{i}$ and $y_{i}$ are the coordinates of node $i, f_{i}$ is the absolute value of the distance error between beacon node $i$ to particle $a$ and unknown node $j, \eta=1 /$ hop $_{i}$ represents weight values for each anchor node, and fitness $(x, y)$ is the particle fitness function. The detailed pseudo-code of the proposed algorithm, which is based on EHP and PSO, is described in Table 1.

\section{Experimental results and discussions}

In this section, the performances of the proposed improved EHP algorithm (proposed) and the PSO positioning algorithm (proposed-PSO) are evaluated by building a simulator in Matlab 2016(a). The performance baseline is given by Amorphous [11], DV-Hop [10], improved EHP [24] and the corresponding DV-maxHop [20] algorithm for comparison under the same network settings. In all simulations, nodes are uniformly deployed in a 2-D area $S=100 \times 100 \mathrm{~m}^{2}$. We consider an anisotropic 
Table 1 Pseudo code of localization algorithm

Algorithm 1 Proposed localization algorithm for multi-hop AWSN.

Input: initialization $N, N_{a}$, anchor node coordinates $\left(x_{i}, y_{i}\right)$, all sensor nodes' communication radius $R$, the size of swarm $M$, the maximum number of iterations $t_{\max }$.

Output: unknown node coordinates $\left(x_{j}, y_{j}\right)$.

1: for $i=1,2, \ldots, N_{a}$ do

2: $\quad$ for $j=N-N_{a}, \ldots, N$ do

3: $\quad$ Employ hops threshold MaxHop to screen effective anchor nodes

4: Compute the distance $d_{i-j}$ of unknown nodes to reliable anchor nodes by equation (1);

5: $\quad$ end for

6: end for

7: Calculate the fitness function value for each particle by equation (13);

8: Compute the values of pbest and gbest;

9: for $t=1,2, \ldots, t_{\max }$ do

10: for $j=1,2, \ldots, M$ do

11: Update position of the particle;

12: Compute fitness by equation (16);

13: $\quad$ if $\operatorname{pbest}(t)<\operatorname{pbest}(t-1)$

14: Update pbest;

15: end if

16: end for

17: Compute gbest;

18: if $\operatorname{gbest}(t)<\operatorname{gbest}(t-1)$

19: Update gbest;

20: end if

21: end for

22: \% take gbest as unknown node coordinates $\left(x_{j}, y_{j}\right)$.

topology that is commonly used in the context of WSNs, namely, the C-shaped topology, as shown in Fig. 1. In addition, we examine the performance of the algorithm in the isotropic network. We always assume that the number of anchors $N_{a}$ is set to 20 and that the total number of nodes $N$ is set to $100,200, \ldots, 500$, and 600 . The transmission capability of $R=20 \mathrm{~m}$ is the same across the network. All simulation parameters are summarized in Table 2, and the simulation results are obtained by averaging over 100 trials.

Table 2 Simulation parameters

\begin{tabular}{ll}
\hline Parameter & Value \\
\hline$S$ & $100 \times 100 \mathrm{~m}^{2}$ \\
$N_{a}$ & 20 or 15:5:45 \\
$\lambda$ & $0.01: 0.01: 0.06$ \\
$r_{i}$ & $20 \mathrm{~m}$ \\
$M$ & 50 \\
$t_{\max }$ & 100 \\
\hline
\end{tabular}

To evaluate the performance of the proposed algorithm, three metrics are utilized, which are defined as follows:

- Localization error

The localization error is the sum of the distances between the nodes' actual positions $\left(x_{j}, y_{j}\right)$ and estimated positions $\left(\hat{x}_{j}, \hat{y}_{j}\right)$, divided by the product of the number of location nodes $N$ and the communication radius $R$. It represents the position estimation deviation with respect to the transmission range, and we use the normalized root-mean-square error (NRMSE), which is defined as follows:

$$
\mathrm{NRMSE}=\frac{\sum_{j=1}^{N} \sqrt{\left(x_{j}-\hat{x}_{j}\right)^{2}+\left(y_{j}-\hat{y}_{j}\right)^{2}}}{N \times R}
$$


The size of the distance error directly affects the localization error. The distance error is the difference between the actual distance and the estimated distance between the two nodes, which can be represented by the mean distance error (MDE) and the distance estimation error (DER), as defined in formula (15) and formula (16), respectively.

$$
\begin{aligned}
& \mathrm{MDE}=\frac{\sum_{i=1}^{N_{a}} \sum_{j=1}^{N_{L}}\left|d_{i-j}-\hat{d}_{i-j}\right|}{N_{a} \cdot N_{L}} \\
& \mathrm{DER}=\frac{\left|d_{i-j}-\hat{d}_{i-j}\right|}{d_{i-j}}
\end{aligned}
$$

where $N_{a}$ is the total number of anchor nodes; $\hat{d}_{i-j}$ and $d_{i-j}$ are the estimated distance and actual distance, respectively, between anchor node $i$ and unknown node $j$. MDE refers to the average distance error from node $i$ to $j$, and DER indicates the relative error between the estimated distance and the true distance.

\section{- Localization percentage}

Due to the hop threshold limit, the percentage of unknown nodes for successful positioning cannot be $100 \%$ because this would result in the absence of anchor nodes. However, the percentage of positioning nodes is an important index for evaluating the localization algorithm. The localization percentage can be expressed as follows:

$$
\text { localizable percentage }=\frac{N_{L}}{N_{u}} \times 100 \%
$$

\subsection{Select the appropriate value of MaxHop}

For the successful positioning of all nodes, the threshold MaxHop should be as close as possible to the smallest integer value. This will not only improve the positioning accuracy but also reduce the computational and communication overheads. However, according to formula (10), the calculated values of MaxHop are often too small. Figure 3 illustrates that the localization nodes' percentage increases with an increasing hop threshold MaxHop. This is because the larger the hop threshold MaxHop is, the more reliable the selected anchor nodes are. When enough anchor nodes are used to locate an unknown node, the probability of the unknown node being successfully positioned increases. According to this figure, when MaxHop is equal to 3, the localization node percentage is close to $100 \%$, regardless of whether the number of anchor nodes in the network is set to 20 or 30. Therefore, we should appropriately increase MaxHop to meet the positioning requirements. In our experiments, we always choose the value of MaxHop to be 3 .

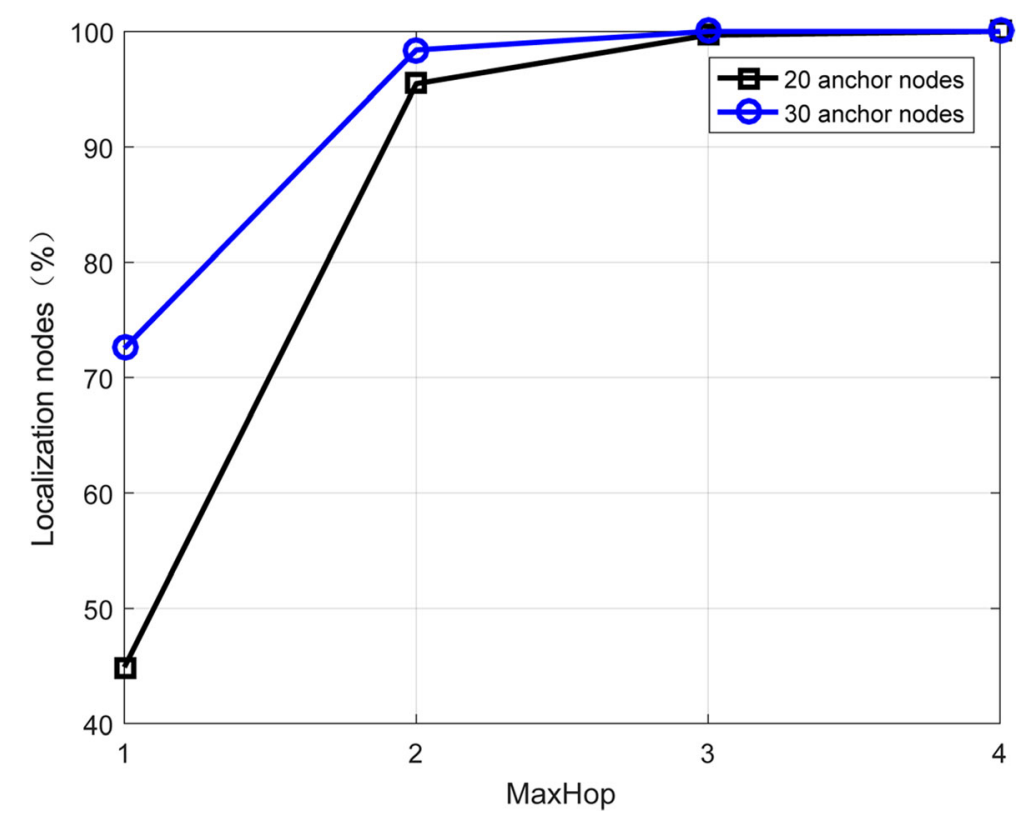

Fig. 3 The influence of MaxHop on localization percentage 


\subsection{Comparison of MDE and NRMSE with various node densities}

Figure 4 shows the localization MDEs achieved by Amorphous, DV-Hop, improved EHP, DV-maxHop, proposed and proposed-PSO for various node densities. The MDE decreases as the node connectivity increases. According to this figure, the results demonstrate that the MDE of the proposed algorithm is less than those of its counterparts under the same network settings. However, when the node density is low, the DV-maxHop algorithm outperforms our algorithm. The MDE of the proposed and proposed-PSO algorithms are approximately the same because the two algorithms use the same method to calculate the distance between nodes. In determining the location of a node, the proposed algorithm uses maximum likelihood estimation, and the proposed-PSO algorithm uses the PSO algorithm.

Next, we discuss the localization errors of the proposed algorithm and other algorithms under different node densities. According to Fig. 5, the proposed algorithm, with or without the PSO algorithm, always outperforms its counterparts. Our proposed algorithm is approximately two, four, and five times more accurate than DV-maxHop, improved EHP, DV-Hop, and Amorphous, respectively. Furthermore, from this figure, the NRMSE that is achieved by the proposed algorithm slightly decreases and then quickly saturates when the node density $\lambda$ increases compared to other algorithms. This is expected since the approximation in formula (1) is more realistic when $\lambda$ is large.
Hence, more accurate localization is achieved. At smaller node densities, the algorithm can also obtain satisfactory results. This indicates that this algorithm can achieve accurate positioning at smaller anchor node density, thereby reducing the localization cost of wireless sensor nodes. This further demonstrates the efficiency and suitability of the proposed localization algorithm in AWSNs.

\subsection{Influence of the number of anchor nodes and iteration times}

In anisotropic networks, the node density is set to 0.06 , and the communication radius is set to 20. Figure 6 shows the NRMSE achieved by Amorphous, DV-Hop, improved EHP, DV-maxHop, proposed and proposedPSO when the number of anchor nodes is varied from 15 to 45 . When the number of anchor nodes is less than 15 (the density of the anchors is less than 0.0015), the percentage of regular nodes for successful positioning cannot be $100 \%$ due to the absence of anchor nodes. The NRMSE achieved by the proposed algorithms significantly decreases initially and then decreases slightly when the number of anchor nodes increases. From the figure, the proposed-PSO algorithm outperforms the traditional algorithms in terms of accuracy.

Figure 7 illustrates the energy consumption and localization NRMSE with different iteration numbers. We can see that with iterations increasing, the PSO algorithm can converge quickly to achieve higher accuracy in multi-hop AWSN localization. On the other hand, as

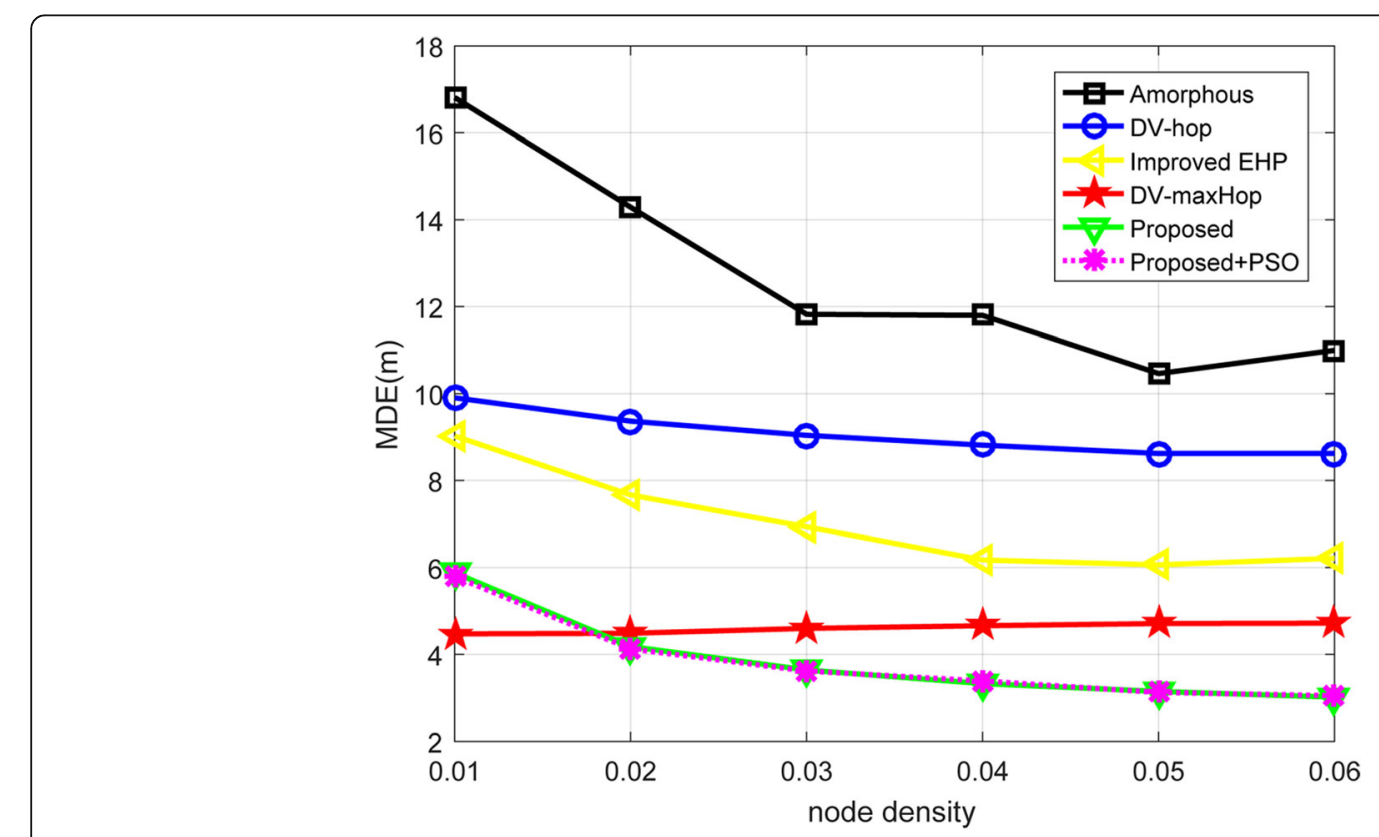

Fig. 4 The influence of node density on MDE 


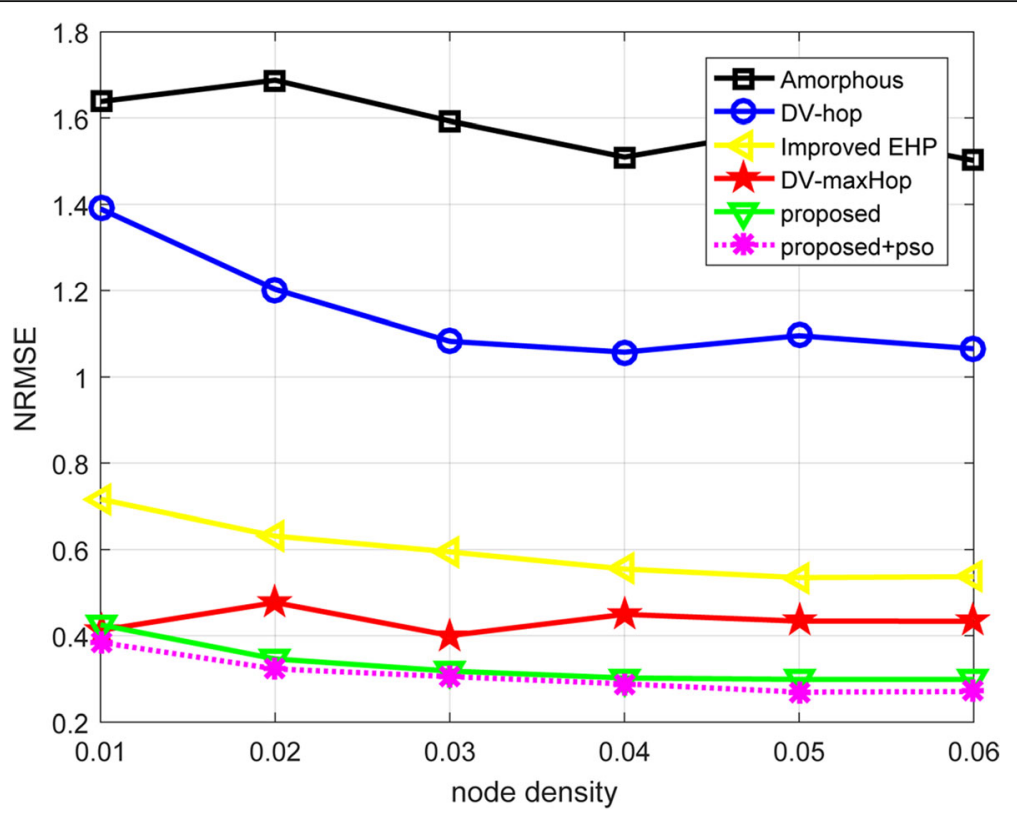

Fig. 5 NRMSE vs. node density in AWSN

the number of iterations increases, so does the runtime, which means that the algorithm consumes more energy. Therefore, the faster convergence can enable us to save the searching overhead.

\subsection{Comparison of localization NRMSE CDFs of various localization methods}

In Fig. 8, we first evaluated the localization results of the proposed algorithms by comparing them to those of
DV-maxHop, improved EHP, DV-Hop and Amorphous by utilizing cumulative distribution function plots. Using the proposed algorithm, $87 \%$ (90\% with proposed-PSO) of the regular nodes could estimate their positions within almost a fifth of their transmission capabilities. In contrast, $67 \%$ of the nodes achieved the same accuracy as DV-maxHop, approximately $55 \%$ as improved EHP, approximately $40 \%$ as DV-Hop, and only $28 \%$ as Amorphous. This further demonstrates the efficiency of

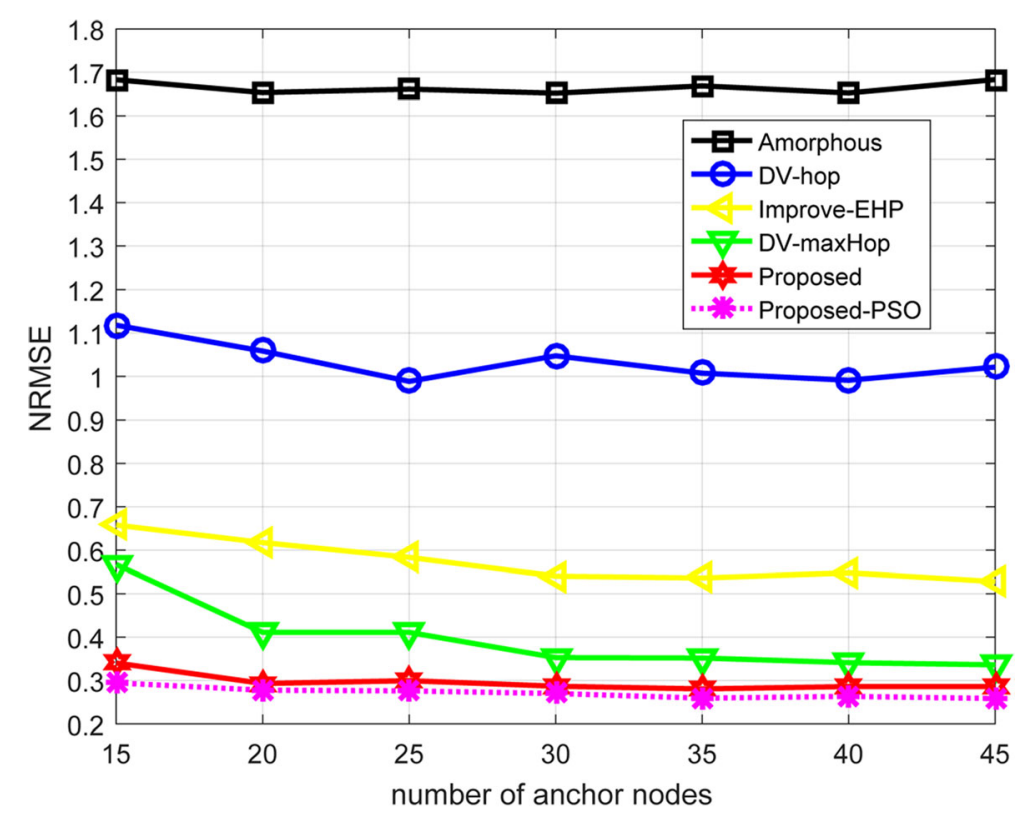

Fig. 6 NRMSE vs. number of anchor nodes in AWSN 


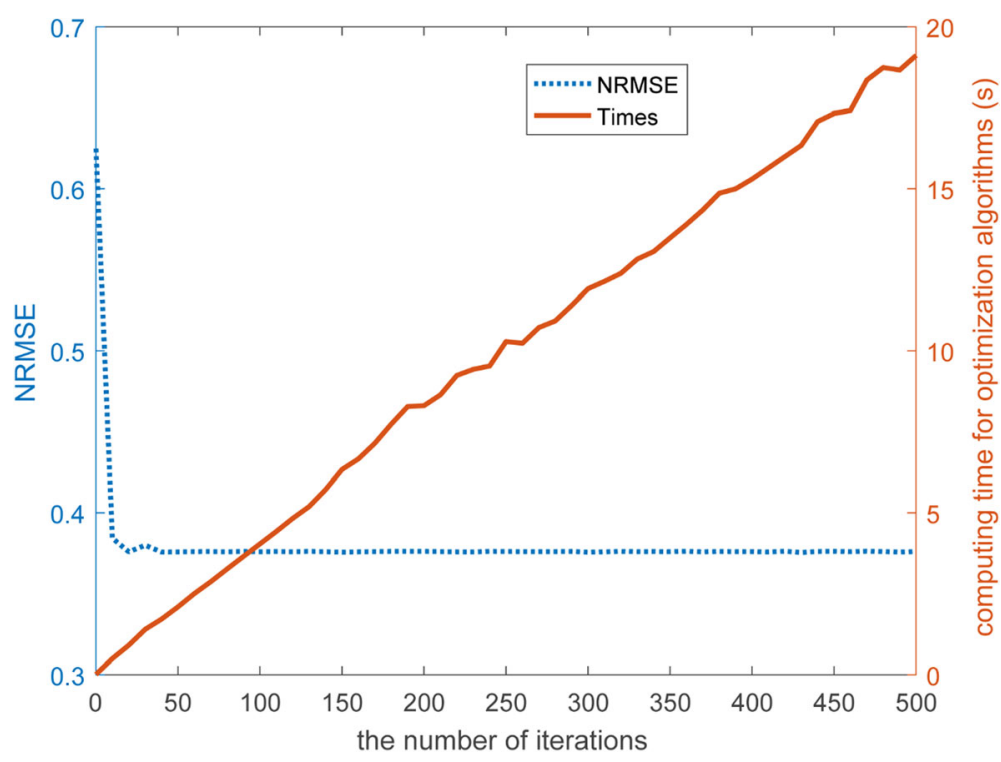

Fig. 7 The energy consumption and convergence analysis of PSO when $\lambda=0.01$

the proposed localization algorithm and indicates that the proposed algorithm is more suitable for AWSNs.

\subsection{Precision analysis of the algorithms in an isotropic WSN}

The algorithm we proposed above is also the most accurate in isotropic WSNs in which the nodes are uniformly deployed in a 2-D square area $S=100 \times 100 \mathrm{~m}^{2}$.
Under the same network settings but a different network topology, we have also carried out a series of experimental studies. Figure 9 plots the localization NRMSEs achieved by Amorphous, DV-Hop, improved EHP, DV-maxHop and our proposed algorithms. According to this data, the localization error of the proposed-PSO algorithm is small in isotropic WSNs and the positioning accuracy is greatly increased in the middle square area $S$

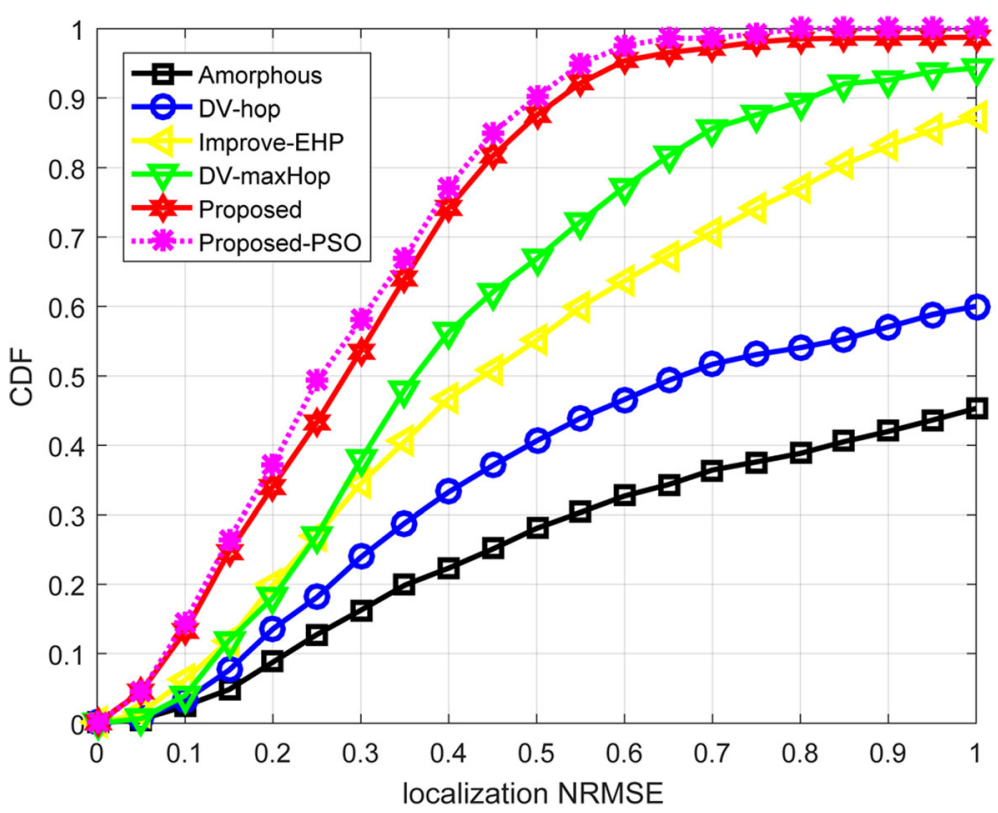

Fig. 8 Localization NRMSE's CDF for different localization methods in AWSN 

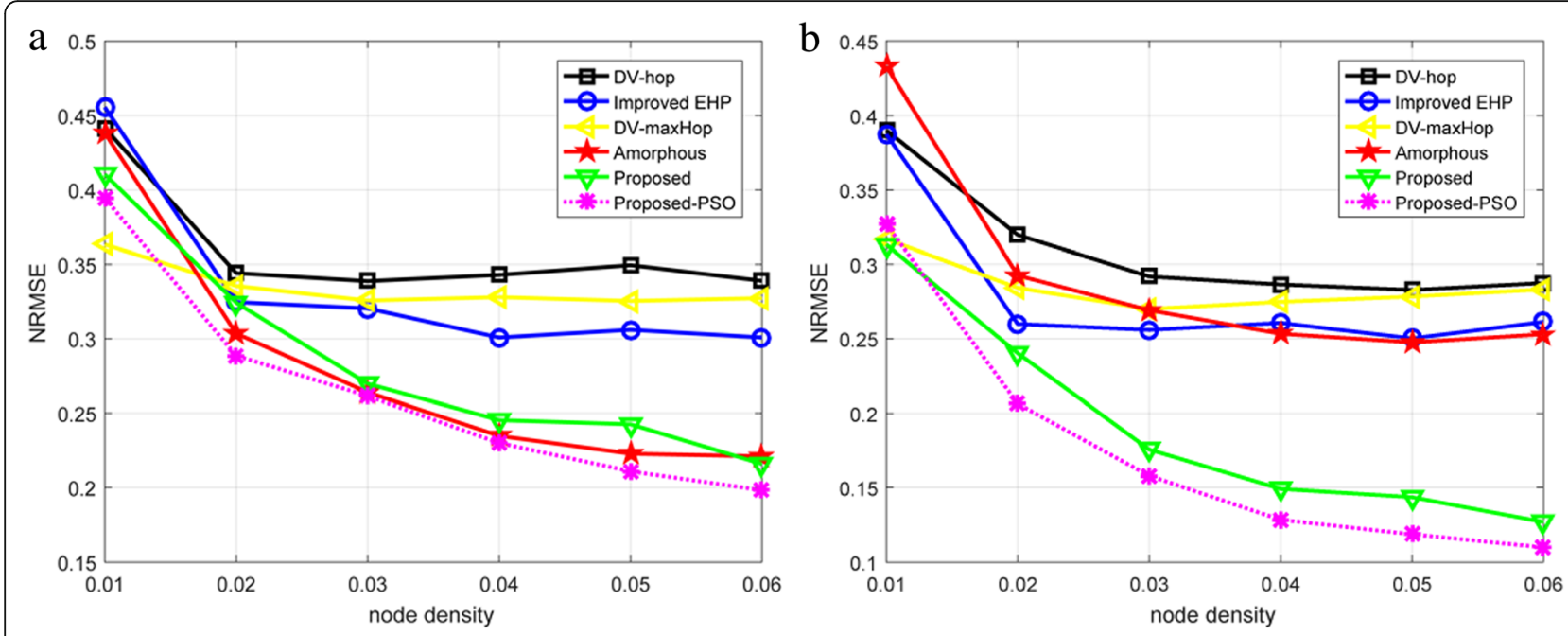

Fig. 9 The influence of node density on NRMSE: a $S=100 \times 100 \mathrm{~m}^{2} ; \mathbf{b} S=90 \times 90 \mathrm{~m}^{2}$

$=90 \times 90 \mathrm{~m}^{2}$. This is because the approximation in formula (1) of the nodes in the middle of the region becomes more realistic, which makes position estimation more accurate. In Fig. 10, the NRMSEs of the two maps are 0.2013 and 0.1081 . The localization accuracy in Fig. 10b is higher than that in Fig. 10a.

Figure 11 shows the localization NRMSE CDF under a node density of 0.06 for square area $S=100 \times 100 \mathrm{~m}^{2}$ and square area $S=90 \times 90 \mathrm{~m}^{2}$. According to this figure, the localization NRMSE CDF of Fig. 11b is far greater than that of Fig. 11a overall. Figure 11b shows that the NRMSE CDF of the proposed-PSO algorithm in the interval of length 0.3 is 99.49 . This result indicates that the localization NRMSE of all nodes is less than 0.3.
This further demonstrates that our algorithm is the most accurate in isotropic WSNs, especially in the middle area $S=90 \times 90 \mathrm{~m}^{2}$.

\section{Conclusion}

In this paper, a novel range-free localization algorithm for multi-hop anisotropic wireless sensor networks is presented. The simulation result demonstrates that our modified algorithm has higher localization precision compared with state-of-the-art algorithms. In addition, our algorithm has achieved good results in isotropic WSNs, especially in the middle of the location area. In general, whether applied with or without the PSO algorithm, our proposed algorithm always outperforms the
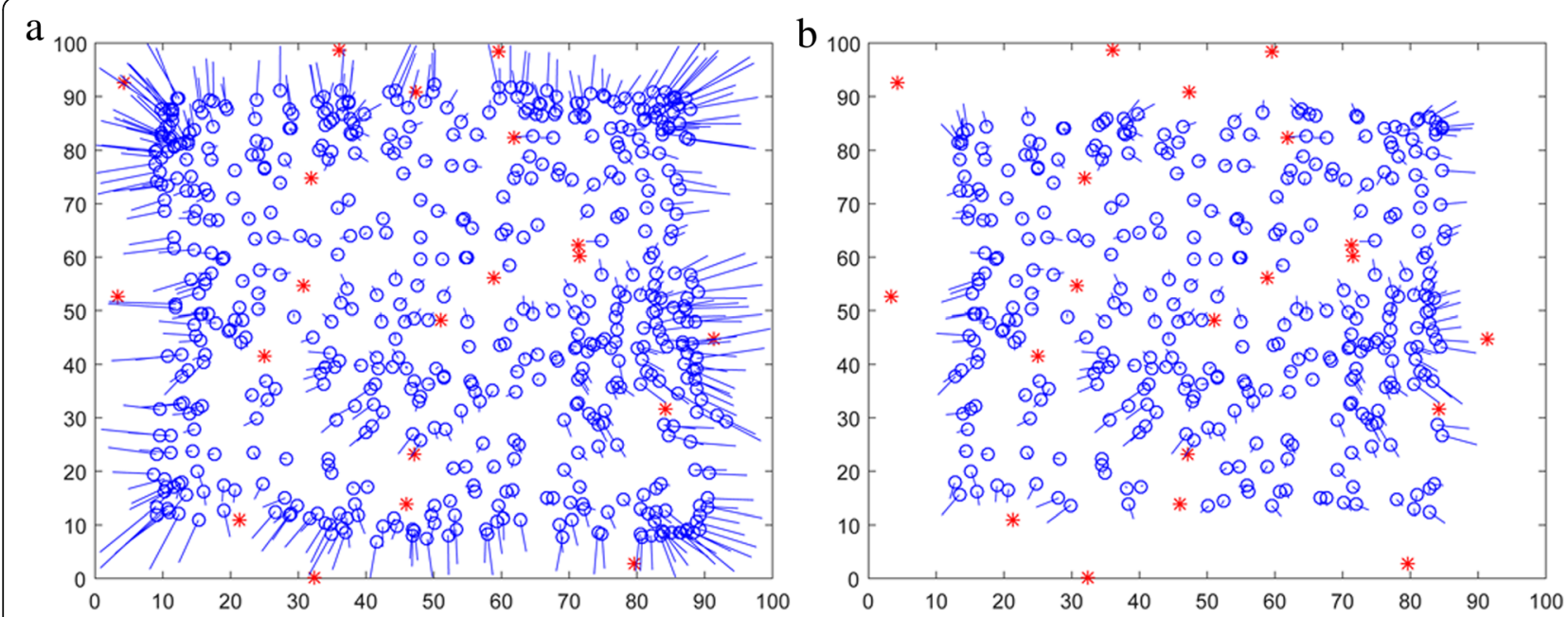

Fig. 10 Node position deviation in isotropic WSN: a $S=100 \times 100 \mathrm{~m}^{2} ; \mathbf{b} S=90 \times 90 \mathrm{~m}^{2}$ 

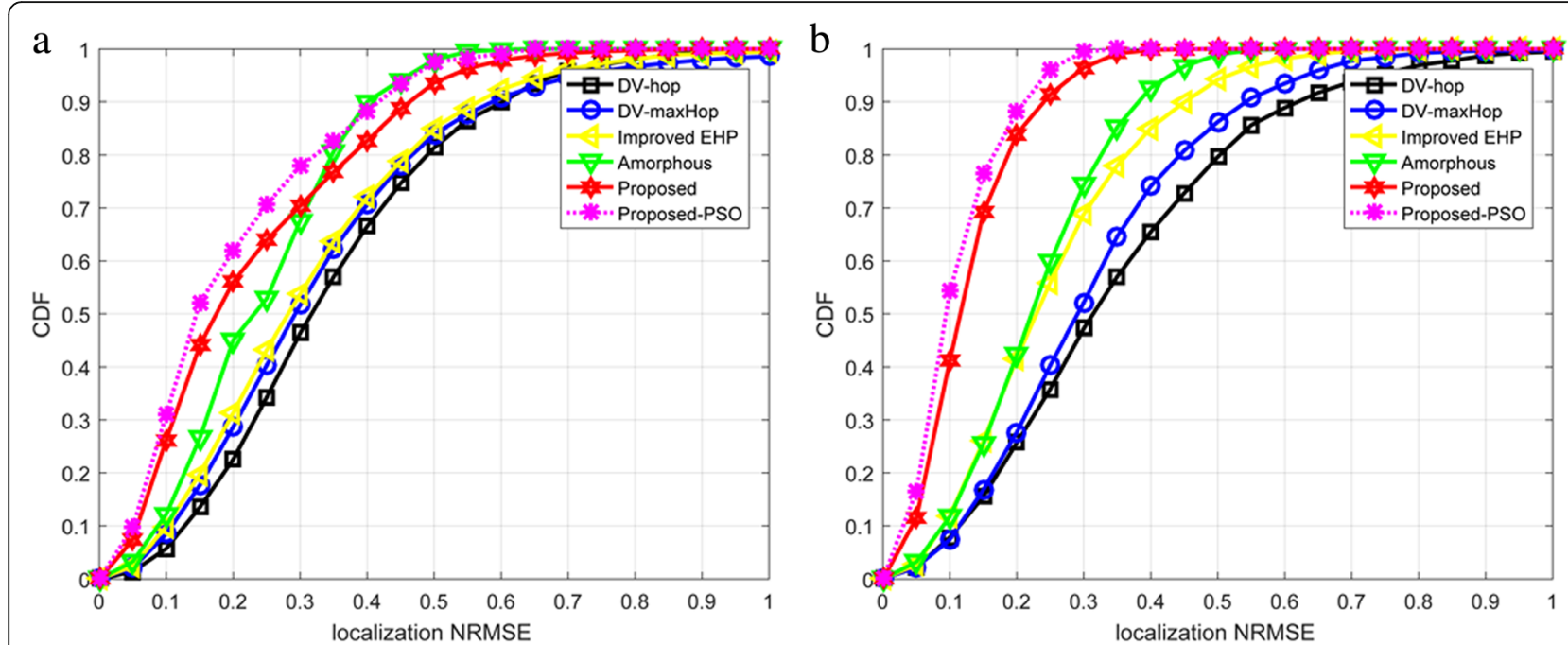

Fig. 11 Localization NRMSE's CDF for different localization methods in AWSN: a $S=100 \times 100 \mathrm{~m}^{2}$; b $S=90 \times 90 \mathrm{~m}^{2}$

most representative WSN localization algorithms. However, if the optimization algorithm is added, the energy consumption will increase while increasing the localization accuracy. As a future work, we plan to study the heterogeneous wireless sensor networks where all nodes' communication ranges are different and we are also planning to study the influence of the irregular communication range in AWSN.

\section{Abbreviations}

2-D: Two-dimensional; ANs: Anchor nodes; AWSN: Anisotropic wireless sensor network; CDF: Cumulative distribution function; DER: Distance estimation error; DOI: Degree of irregularity; EHP: Expected hop progress; GPS: Global positioning system; LAEP: Localization algorithm using expected hop progress; MDE: Mean distance error; NRMSE: Normalized root-mean-square error; PSO: Particle swarm optimization; UNs: Unknown nodes; WSN: Wireless sensor network

\section{Acknowledgements}

Not applicable.

\section{Funding}

This work is supported by National Natural Science Foundation of China (No. 61472278 and 61102125), Key project of Natural Science Foundation of Tianjin University (2017ZD13), the Research Project of Tianjin Municipal Education Commission (No. 2017KJ255).

\section{Availability of data and materials}

Not applicable.

\section{Authors' contributions}

WW proposed the main idea and performed the simulation and manuscript writing. XW provided guidance for the algorithm design and helped revise the manuscript. All authors read and approved the final manuscript.

\section{Authors' information}

Wu Wen received his bachelor's degree in communication engineering from Liaoning University of Technology in 2016. He is pursuing her master's degree in communication and information engineering at Tianjin University of Technology, China. His research interests include anisotropic wireless sensor networks, wireless sensor networks localization and performance evaluation and optimization.

Xianbin Wen received his PhD from the Northwestern Polytechnical University, Xi'an, China, in 2005. He is currently a professor with the School of
Computer and Communication Engineering, Tianjin University of Technology, Tianjin, China. His research interests include image interpretation, machine learning, and information hiding. Liming Yuan received the PhD degree in computer science and technology from Harbin Institute of Technology, China, in 2014. He is currently working as a lecturer in the School of Computer Science and Engineering at Tianjin University of Technology, China. His research interests are mainly in machine learning and image processing.

Haixia Xu received her MSc degree in applied mathematics from the Northwestern Polytechnical University, China, in 2006, and a PhD in computer science and technology from the same university in 2009. She is currently an associate professor at the School of Computer and Communication Engineering, Tianjin University of Technology, China. Her main research interests include image analysis, signal processing, and pattern recognition.

\section{Competing interests}

The authors declare that they have no competing interests.

\section{Publisher's Note}

Springer Nature remains neutral with regard to jurisdictional claims in published maps and institutional affiliations.

\section{Author details}

${ }^{1}$ Key Laboratory of Computer Vision and System, Ministry of Education, Tianjin University of Technology, Tianjin 300384, China. ${ }^{2}$ Tianjin Key Laboratory of Intelligence Computing and Novel Software Technology,

Tianjin University of Technology, Tianjin 300384, China.

Received: 14 May 2018 Accepted: 11 December 2018

Published online: 27 December 2018

\section{References}

1. Y. Zhu, Y. Zhang, W. Xia, L. Shen, in A Software-Defined Network Based Node Selection Algorithm in WSN Localization. 83rd vehicular technology conference (Nanjing, 2016), pp. 1-5

2. H. Zheng, W. Guo, N. Xiong, A kernel-based compressive sensing approach for mobile data gathering in wireless sensor network systems. IEEE Trans. Syst. Man Cybern. Syst. 48(12), 1-13 (2017)

3. N. Xiong, X. Jia, L. Yang, A. Vasilakos, Y. Li, Y. Pan, A distributed efficient flow control scheme for multirate multicast networks. IEEE Trans. Parallel Distrib. Syst. 21(9), 1254-1266 (2010)

4. B. Lin, W. Guo, N. Xiong, G. Chen, A. Vasilakos, H. Zhang, A pretreatment workflow scheduling approach for big data applications in multi-cloud environments. IEEE Trans. Netw. Serv. Manag. 13(3), 581-594 (2016) 
5. S. Tomic, M. Beko, R. Dinis, M. Tuba, N. Bacanin, in An efficient WLS estimator for target localization in wireless sensor networks. 24th telecommunications forum (Belgrade, 2017), pp. 1-4

6. B. Xiao, L. Chen, Q. Xiao, Reliable anchor-based sensor localization in irregular areas. IEEE Trans. Mob. Comput. 9(1), 60-72 (2010)

7. L.Z. Zhao, X.B. Wen, D. LI, Amorphous localization algorithm based on BP artificial neural network. Int. J. Distrib. Sens. Netw (2015). https//doi.org/10.1155/2015/657241

8. T. Alhmiedat, A.A. Salem, A hybrid range-free localization algorithm for ZigBee wireless sensor networks. Int. Arab J. Inf. Technol 14(4A), 647-653 (2017)

9. N.B.M. Ngabas, J.B. Abdullah, in Comparison of energy consumption in cooperative range-free localization for wireless sensor network. IEEE student conference on research and development (Putrajaya, 2013), pp. 459-464

10. Q.G. Gao, L. Lei, in 2nd International Conference on Advanced Computer Control An improved node localization algorithm based on DV-HOP in WSN (Shenyang, 2010), pp. 321-324

11. S. Shen, B. Yang, K. Qian, W. Wang, X. Jiang, Y. She, Y. Wang, in An improved amorphous localization algorithm for wireless sensor networks. International conference on networking and network applications (Hakodate, 2016), pp. 69-72

12. X. Sun, T. Chen, W. Li, M. Zheng, in International Conference on Computer Science and Electronics Engineering Perfomance research of improved MDS-MAP algorithm in wireless sensor networks localization (Hangzhou, 2012), pp. 587-590

13. Y Zhou, X Ao, S Xia, in 7th World Congress on Intelligent Control and Automation. An improved APIT node self-localization algorithm in WSN, (Chongqing, 2008), pp. 7582-7586

14. H. Lim, J.C. Hou, in Localization for anisotropic sensor networks. 24th annual joint conference of the IEEE computer and communications societies (Miami, 2005), pp. 138-149

15. Q. Xiao, B. Xiao, J. Cao, J. Wang, Multihop range-free localization in anisotropic wireless sensor networks: a pattern-driven scheme. IEEE Trans. Mob. Comput. 9(11), 1592-1607 (2010)

16. X. Liu, S.G. Zhang, K. Bu, A locality-based range-free localization algorithm for anisotropic wireless sensor networks. Telecommun. Syst 62(1), 3-13 (2016)

17. Y. Wang, X.D. Wang, D.M. Wang, D.P. Agrawal, Range-free localization using expected hop progress in wireless sensor networks. IEEE Trans. Parallel Distrib. Syst 20(10), 1540-1552 (2009)

18. AE Assaf, S Zaidi, S Affes, N Kandil, in IEEE International Conference on Communications (ICC). Cost-effective and accurate nodes localization in heterogeneous wireless sensor networks. (London, 2015), pp. 6601-6608

19. A.E. Assaf, S. Zaidi, S. Affes, N. Kandil, Low-cost localization for multihop heterogeneous wireless sensor networks. IEEE Trans. Wirel. Commun. 15(1), 472-484 (2016)

20. F. Shahzad, T. Shaltami, E. Shakshukhi, DV-maxHop: a fast and accurate range-free localization algorithm for anisotropic wireless networks. IEEE Trans. Mob. Comput. 16(9), 2494-2505 (2017)

21. $\mathrm{F}$ Zhou, $\mathbf{S}$ Chen, in Proceedings of the 2016 International Conference on Communications. DV-Hop Node Localization Algorithm Based on Improved Particle Swarm Optimization, (2016), pp. 541-550

22. W. WL, X.B. Wen, H.X. Xu, L.M. Yuan, Q.X. Meng, Efficient range-free localization using elliptical distance correction in heterogeneous wireless sensor networks. Int. J. Distrib. Sens. Netw. (2018). https://doi.org/10.1177/ 1550147718756274

23. W. WL, H.X.X. XB Wen, L.M. Yuan, Q.X. Meng, Accurate range-free localization based on quantum particle swarm optimization in heterogeneous wireless sensor networks. KSII Trans. Internet Inf. Syst. 12(3), 1083-1097 (2018)

24. A.E. Assaf, S. Zaidi, S. Affes, N. Kandil, in Accurate Nodes Localization in Anisotropic Wireless Sensor Networks. IEEE International Conference on Ubiquitous Wireless Broadband (ICUWB) (2015), pp. 1-5

25. A.E. Assaf, S. Zaidi, S. Affes, Robust ANNs-based WSN localization in the presence of anisotropic signal attenuation. IEEE Wirel. Commun. Lett 5(5), 504-507 (2016)

26. S Zaidi, AE Assaf, S Affes, N Kandil, in IEEE International Conference on Ubiquitous Wireless Broadband. Range-Free Nodes Localization in Mobile Wireless Sensor Networks, (2015), pp. 1-6

27. Y. Yue, L. Ding, H. Zhao, H. Wang, in IEEE International Conference on Mechatronics and Automation (ICMA Study on DV-HOP node location algorithm for Wireless Sensor Networks (Takamatsu, 2017), pp. 1709-1713

28. R. Zhang, F. Yan, W. Xia, S. Xing, Y. Wu, L. Shen, An Optimal Roadside Unit Placement Method for VANET Localization (Singapore, 2017), pp. 4-8. IEEE Global Communications Conference

29. W. Guo, N. Xiong, A. Vasilakos, G. Chen, C. Yu, Distributed k-connected fault-tolerant topology control algorithms with PSO in future autonomic sensor systems. Int. J. Sens. Netw. 12(1), 53-62 (2012)

\section{Submit your manuscript to a SpringerOpen ${ }^{\circ}$ journal and benefit from:}

- Convenient online submission

- Rigorous peer review

- Open access: articles freely available online

High visibility within the field

- Retaining the copyright to your article

Submit your next manuscript at $\boldsymbol{\nabla}$ springeropen.com 